\title{
Guest Associate Editors
}

Hank Schlinger, California State University, Los Angeles

Tina Sidener, Caldwell College

\section{Guest Reviewers}

Chata Dickson, New England Center for Children and E. K. Shriver Center Kate Endicott-Harris, Utah State University

Amanda Guld, Melmark

Carl Hughes, Bangor University, Wales

Kent Johnson, Morningside Academy

Michael Kelley, University of Southern Maine

Danielle LaFrance, B.E.S.T. Consulting, Inc.

T. V. Joe Layng, Headsprout

Harry Mackay, Northeastern University and Praxis, Inc.

Amanda Mahoney, Western Michigan University

Pat McGreevy, Florida Institute of Technology

Charles Merbitz, The Chicago School of Professional Psychology

Ed Morris, University of Kansas

Martha Pelaez, Florida International University

Bob Remington, University of Southampton, United Kingdom

Rocio Rosales, Youngstown State University

Daniel Shabani, California State University, Los Angeles

Tim Slocum, Utah State University

Rachel Thompson, Western New England College

Jeffrey Tiger, Louisiana State University

Manish Vaidya, University of North Texas

J. Virues-Ortega, Centro Nacional de Epidemiología, Instituto de Salud Carlos III, Spain

Ray Weitzman, California State University, Fresno 